\title{
Traumatic Perforation of a Pacemaker Generator into the Abdominal Cavity
}

\begin{abstract}
Keywords: Pacemaker, Generator migration; Epic ardial pacemaker
\section{Abstract}

We report a case of a pacemaker generator of an epicardia system migrated into the abdominal cavity due to a traffic accident and its subsequent retrieval with a laparoscopic technique in an adult patient. The retrieval of the dislocated pacemakerproceeded without any incident. The pacemaker generator was placed in a parastemal subpectoral pocket on the left chest wall, which makes another perforation highly impossible.
\end{abstract}

\section{Case Report}

A 57-year-old male patient was referred to our trauma department after a pedestrian-motor vehicle accident. The patient had a history of epicardial dual chamber pacemaker implantation due to a third degree AV-block post aortic valve replacement. On arrival he was well orientated and presented with a Glasgow Coma Scale of 15. Due to a continued drug abuse he was in a reduced state of general health. His left hemithorax showed a contusion. The routine trauma CT scan revealed a trochanteric fracture and a fracture of the pelvic ring. As an incidental finding a dislocation of the pacemaker generator into the abdominal cavity was diagnosed (Figure 1).

In the past the patient had received an aortic valve replacement due to aortic valve endocarditis with severe aortic valve regurgitation. He needed a redo aortic valve replacement 6 months later due to endocarditis of the prosthetic valve. After the second aortic valve procedure a third degree AV block occurred which necessitated dual chamber pacemaker implantation. This was performed epicardially due to the history of endocarditis. At that time the pacemaker

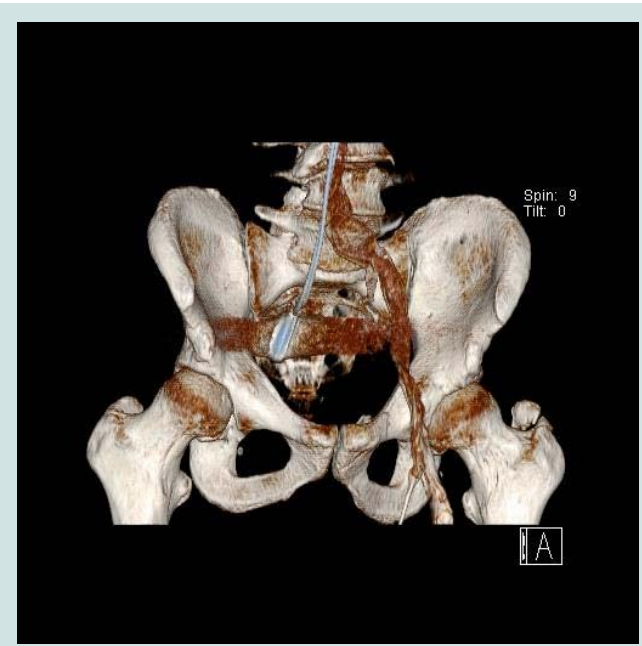

Figure 1: CT scan showing the dislocated pacemaker in the true pelvis

\section{Journal of}

\section{Cardiobiology}

Salomon Manz ${ }^{1}$, Felix Schoenrath², Volkmar Falk², Francesco Maisano ${ }^{1}$ and Christoph T. Starck ${ }^{2 *}$

${ }^{1}$ Clinic of Cardiac and Vascular Surgery, University Hospital Zurich, Switzerland

${ }^{2}$ Department of Cardiothoracic and Vascular Surgery, German Heart Institute Berlin, Germany

*Address for Correspondence

PD Dr. Christoph T. Starck, Department of Cardiothoracic and Vascular Surgery, German Heart Institute Berlin, Augustenburger Platz 1, 13353

Berlin, Germany, E-mail: starck@dhzb.de

Submission: 23 July, 2015

Accepted: 31 July, 2015

Published: 04 August, 2015

Copyright: $\odot 2015$ Manz S, et al. This is an open access article distributed under the Creative Commons Attribution License, which permits unrestricted use, distribution, and reproduction in any medium, provided the original work is properly cited.

Reviewed \& Approved by: Dr. Krasniqi Nazmi, Division of Cardiology, University Hospital of Zurich, Switzerland

generator was placed behind the rectus muscle in front of the posterior rectus sheath.

The retrieval-procedure of the dislocated pacemaker was started as a diagnostic laparoscopy in order to assess the local situation and to gain further information on the precise locations of adjacent organs. The pacemaker was identified in the pelvis without extensive adhesions or damage to adjacent organs. Based on this information, it was decided to perform a laparoscopic retrieval of the pacemaker. The pacemaker generator was successfully removed and due to its size externalized through a mini-laparotomy. The pacemaker leads had to be freed from the bowel loops and subsequently were also externalized through the mini-laparotomy. In order to avoid another perforation the pacemaker was placed on the chest wall behind the left pectoral muscle. The patient recovered uneventful from this procedure.

One week later, the patient underwent an orthopedic surgery because of the femur fracture, as this was defined second priority upon referral. Seven days later, the patient was discharged from the hospital.

\section{Discussion}

Patients, in whom the transvenous route is not accessible or inappropriate, may receive epicardial pacemaker leads with the device subsequently placed in a generator pocket located in the abdominal wall [1-3]. Furthermore patients undergoing cardiac surgical procedures with concomitant need of pacemaker therapy and patients with tricuspid valve prosthesis may receive epicardial pacemaker systems at the time of the initial operation [4].

In addition to locating the pacemaker generator into the abdominal wall (within the rectus sheath behind the muscle) [1], subclavicular positioning or inserting the generator in a pocket on the chest wall is possible. In the reported case the patient had an epicardial pacemaker system with the device placed behind the rectus muscle. 


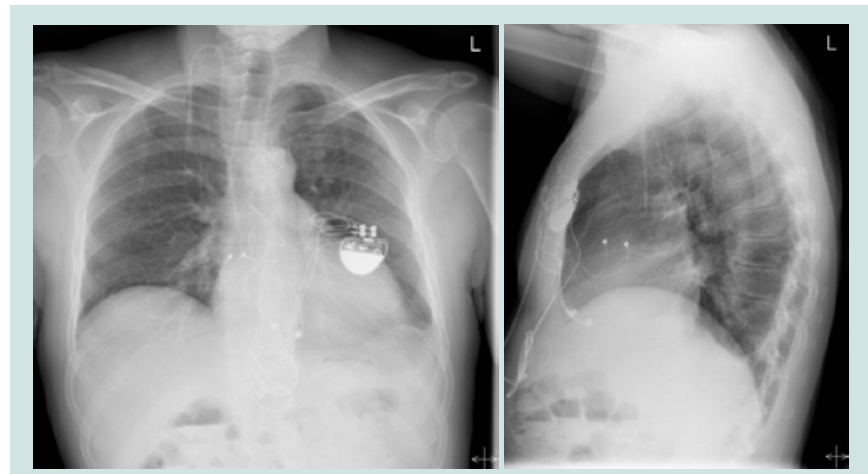

Figure 2: Postoperative chest x-ray demonstrating the new sub pectoral generator pocket on the left chest wall.

Migration of pacemaker generators, which are placed behind the rectus muscle, is a known but rare complication that is mainly reported in children [1-3]. Based on a thorough literature review only few cases of intraperitoneal migration of epicardial pacemakers were found in the current literature [1].

Pacemaker migration may cause different clinical presentations such as diarrhea, abdominal pain, intestinal obstruction or even life threatening complications like intestinal perforation [5]. Even though the patient did not have any of these symptoms, the dislocation of the pacemaker was diagnosed early with the routine CT in the trauma department.

It is very likely that the trauma caused the perforation because no extensive adhesions of the pacemaker generator or the leads were found during the laparoscopic procedure.

The described laparoscopic retrieval of the pacemaker generator from the true pelvis is a safe and efficient minimal-invasive technique, which has many advantages compared to a laparotomy. Due to the smaller surgical trauma, the patient has a higher chance for a quick recovery, suffers less pain and consequently may be discharged earlier. Additionally, the peri- and postoperative risk of infection is reduced.

The pacemaker generator was subsequently placed in a sub pectoral pocket on the left chest wall as shown in Figure 2. The advantage of this position is that pacemaker generator perforation into the thoracic cavity is impossible, because the ribs prevent any such migration.

\section{Conclusion}

The described laparoscopic approach for the retrieval of an abdominally perforated pacemaker was successful in precisely locating the device, assessing the local situation and safely retrieving the generator and leads. The described novel generator pocket on the left chest wall may be a good alternative for device positioning of epicardial pacemaker systems implanted by a subxiphoidal approach, a partial lower sternotomy or a median sternotomy to avoid the complication of an abdominal generator perforation.

\section{References}

1. García-Bengochea J, Caínzos M, Fernández AL, Santos F, Gonzalez F (2007) Intraperitoneal migration of epicardial pacemakers. Tex Heart Inst J 34: $376-378$

2. Kravarusic D, Chirdan L, Freud E (2011) Laparoscopic-assisted retrieval of migrated intra-abdominal pacemaker generator. Pediatr Cardiol 32: 11961198.

3. Li KS, Khwaja HA, Hayat TT, Asghar A, Alsarakbi W, et al. (2007) A rare cause of abdominal pain. Ann R Coll Surg Engl 89: W14-W16.

4. Lazarescu C, Mertes PM, Longrois D (2013) Temporary epicardial pacing followingcardiac surgery: practical aspects. Ann Fr Anesth Reanim 32: 592601.

5. Charles R, Turner WL (1978) Diarrhoea induced by migration of a pacemaker generator. Br Heart J 40: 425-426. 